Revista Paulista de Enfermagem

Recebido: 23.04.2019

Aceito: 06.08.2019

Como citar este artigo

Silva MSGO, Sá LO, Marques GFS. Impacto do Transtorno Neurocognitivo na Família:

Scoping Review] Rev Paul Enferm

[Internet]. 2019;30 doi:10.33159/25959484 repen.2019v30a4

\title{
Impacto do Transtorno Neurocognitivo na Família: Scoping Review
}

\author{
The Impact of Neurocognitive Disorder in the Family: A Scoping Review \\ Impacto del Transtorno Neurocognitivo en la Familia: Scoping Review
}

\author{
Mafalda Sofia Gomes Oliveira da Silva', Luís Octávio de SáII, \\ Goreti Filipa Silva Marques ${ }^{\text {III }}$
}

I Doutoranda em Enfermagem, Universidade Católica Portuguesa, Instituto de Ciências da Saúde, Porto, Centro de Investigação Interdisciplinar em Saúde; Professora Adjunta, Escola Superior de Saúde de Santa Maria; mafalda.silva@santamariasaude.pt

II Professor Auxiliar, Universidade Católica Portuguesa, Instituto de Ciências da Saúde, Porto, Centro de Investigação Interdisciplinar em Saúde; Isa@porto.ucp.pt

III Professora Coordenadora, Departamento de Enfermagem/Escola Superior de Saúde de Santa Maria (ESSSM), Porto, Portugal; Centro de Investigação em Tecnologias e Serviços de Saúde (CINTESIS) - NursID, Porto, Portugal.

Autora Correspondente

Mafalda Silva Morada

E-mail:

mafalda.silva@ santamariasaude.pt Endereço: Tv. de Antero de Quental 173 175,

4049-024

Porto, Portugal

Tel: 933764837

\section{RESUMO}

Objetivos: Conhecer a literatura científica existente sobre o impacto do aparecimento da demência; identificar os diferentes membros do agregado familiar que têm sido alvo de estudo; identificar as metodologias utilizadas e conhecer as dimensões do impacto da demência na família. Método: Scoping Review inspirada nos princípios preconizados pelo Joanna Briggs Institute. Dois revisores independentes realizaram a análise de relevância dos artigos, a extração e síntese dos dados. Após a seleção, constituiu-se um corpus de análise, composto por 27 estudos. Resultados: Os cuidados às pessoas com demência são assegurados por cuidadores informais, na sua grande maioria mulheres, destacando-se o papel das esposas, filhas e noras. O impacto resultante do surgimento desta doença tem sido amplamente estudado, com incidência, nas repercussões positivas e negativas que advêm do cuidar. Conclusão: Não só o cuidador informal, mas também a restante família têm especial interesse pelo bem-estar, saúde e doença dos seus membros, tornando-se importante envolvê-la como parte integrante da investigação e da prática de cuidados de Enfermagem. Descritores: Demência; Relações familiares; Enfermagem; Adaptação.

\section{ABSTRACT}

Aims: To investigate the existing scientific literature on the impact of dementia; to identify the different household members who have been investigated in studies; to identify the research methods being used and to verify the dimensions of the impact of dementia in the family. Method: This scoping review was 
based on the principles developed by the Joanna Briggs Institute. Two independent reviewers analyzed the papers in terms of relevance, extracted data, and synthesized data. 27 studies were selected. Results: Informal care is provided to patients with dementia mainly by women, and especially by wives, daughters, and daughters-in-law. The impact resulting from the onset of this disease has been widely studied, and it influences caregiving both positively and negatively. Conclusion: The informal caregiver, as well as the whole family, are especially interested in the well-being, health, and disease of their relatives. Therefore, involving caregivers and families in research and nursing care is fundamental.

Keywords: Dementia; Family relations; Nursing; Adaptation.

\section{RESUMEN}

Objetivos: Conocer la literatura científica existente sobre el impacto de la aparición de la demencia e identificar a los diferentes miembros del agregado familiar que han sido objeto del estudio; identificar las metodologias utilizadas y conocer las dimensiones del impacto de la demencia en la família. Método: Scoping Review inspirada en los principios recomendados por el Joanna Briggs Institute. Dos revisores independientes realizaron el análisis de relevancia de los artículos, la extracción y síntesis de los datos. Después de la selección, se constituyó un corpus de análisis, compuesto por 27 estudios. Resultados: Los cuidados a las personas con demencia son asegurados por cuidadores informales, en su gran mayoria mujeres, destacándose el papel de las esposas, hijas y nueras. El impacto resultante del surgimiento de esta enfermedad ha sido ampliamente estudiado, com incidencia, en las repercusiones positivas y negativas que resultan del cuidar. Conclusión: No solo el cuidador informal, sino también el restante de la família tiene un interés especial por el bienestar, salud y enfermedad de sus miembros, por lo que es importante involucrarla como parte integrante de la investigación y de la práctica de cuidados de Enfemería. Descriptores: Demencia; Relaciones familiares; Enfermería; Adaptación.

\section{INTRODUÇÃO}

Verifica-se nas últimas décadas um aumento da esperança média de vida e da melhoria da qualidade de vida, resultante de melhores condições socioeconómicas e dos avanços tecnológicos nos cuidados de saúde. O envelhecimento populacional tem crescido de forma exponencial nos últimos anos, gerando progressivo aumento de doenças crónico-degenerativas, entre elas a demência. Estima-se que existam no mundo cerca de 35,6 milhões de pessoas com esta doença. Segundo a Associação Brasileira de Alzheimer, no Brasil há cerca de 1,2 milhão de casos, na sua maioria sem diagnóstico. Segundo o relatório da Organização para a Cooperação e Desenvolvimento Económico (OCDE) publicado em novembro $2017^{(1)}$, Portugal é considerado o $4^{\circ}$ país com maior número de casos por cada mil habitantes. Estima-se que existam cerca de 205 mil pessoas com demência em Portugal. A média da OCDE é de 14.8 casos por cada mil habitantes e em Portugal a estimativa é de 19.9. Espera-se que até 2037 o número de casos aumente para os 322 mil.

Apesar de em Portugal haver uma política descentralizadora dos cuidados hospitalares, o acesso aos serviços sociais e de saúde são ainda um processo complexo, demorado e tardio na assistência à pessoa com vulnerabilidade. Nas últimas décadas o conceito de família tem adquirido uma maior complexidade, com o surgimento de novas conceções de família, novas dinâmicas familiares, novos valores, diferentes relações, bem como, as alterações no papel da mulher a nível social e familiar. Esta alteração na estrutura e na dinâmica familiar implica que as famílias estejam limitadas para assegurar os cuidados de saúde adequados à pessoa dependente, refletindo-se no aumento da procura de apoio institucional.

O suporte social é uma condição fundamental para a proteção da pessoa contra a deterioração da saúde e bem-estar com a vida(2). É urgente que as políticas de saúde e de serviços sociais resultem em soluções acessíveis aos cidadãos. As demências causam grande impacto 
no seio familiar, aumentando a sobrecarga emocional de todos os membros do agregado familiar, mas principalmente do cuidador e gerando conflitos na dinâmica familiar. $\mathrm{O}$ ato de cuidar é transversal a todas as culturas manifestando-se de diferentes formas. $\mathrm{O}$ exercício do cuidado e a responsabilidade sobre idosos dependentes é um dos papéis familiares, e uma função extremamente desgastante que exige das famílias uma reorganização funcional e a aquisição de novas competências.

Numa perspetiva sistémica, a família pode ser considerada como um sistema aberto. Os subsistemas que a compõem interagem, dentro e fora da família, uns com os outros, e com outros sistemas e subsistemas extrafamiliares. É um processo constante de troca de informação permitindo o seu desenvolvimento e bem-estar, através do intercâmbio entre o sistema familiar e o exterior. Possibilita que cada membro enquanto subsistema da família tenha as suas formas de se dinamizar, realizar tarefas e funções que conduzem ao seu desenvolvimento. Por conseguinte, os comportamentos e ações de um dos seus membros tanto pode influenciar como ser influenciado pelos comportamentos dos outros.

As alterações resultantes do aparecimento de uma doença crónica têm repercussões na família a vários níveis: físico, psíquico, social, económico e na sua dinâmica relacional, diminuindo a independência e autonomia da pessoa doente e, por conseguinte, a qualidade de vida de todos os elementos do agregado familiar ${ }^{(3)}$. Perante os desafios da readaptação familiar que o aparecimento de um quadro demencial provoca, consideramos que os enfermeiros necessitam de aprofundar o conhecimento sobre o impacto/consequências que a demência implica na família, em todas as suas dimensões, para contribuírem no desenvolvimento de uma prática baseada na evidência. A conceção dos cuidados à família assenta no pensamento sistémico, permitindo a compreensão multidimensional, instabilidade, globalidade, contextualidade e organização inerentes ao sistema familiar ${ }^{(3)}$. Olhando através deste paradigma é possível compreender diferentes dinâmicas e contextos familiares que vão para além da família nuclear. Apesar das alterações organizacionais e estruturais que a família tem sofrido ao longo dos tempos, continua a ser uma unidade emocional e afetiva, e porque não dizê-lo, de saúde também, que será eventualmente a unidade de saúde mais estável ao longo do ciclo de vida.

O surgimento no seio familiar de um elemento que precisa de ajuda para a realização das atividades diárias acarreta alterações substanciais não só para si, mas também para a própria família(4).

Assim, com a presença de um familiar doente, a família vai sofrer um conjunto de modificações no dia-a-dia. Para conseguirem dar uma resposta eficaz ao aparecimento de um quadro demencial, torna-se necessária a aquisição de um conjunto de conhecimentos e competências aos diferentes níveis: um plano de desenvolvimento, aprender a lidar com diversas situações e disponibilidade de recursos financeiros, podendo refletir dificuldades em se reorganizarem. As consequências são sentidas por toda a família, sendo necessária uma abordagem global centrada nas suas necessidades biológicas, psicológicas, sociais e espirituais ${ }^{(5)}$.

Neste contexto, é fundamental o papel que os enfermeiros assumem na prestação de cuidados às famílias, de forma, a promover a autonomia e o crescimento do sistema familiar. Cada família possui uma dinâmica própria que lhe concede a individualidade e a sua autonomia estando sujeita a fatores externos, mas também a "forças internas" providenciando a harmonia necessária. Mais especificamente, esta revisão pretende dar resposta às seguintes questões:

Qual a literatura científica existente sobre a adaptação da família face ao aparecimento de um quadro demencial num dos seus membros?

Quais os membros do agregado familiar que tem sido alvo de estudo?

Quais as metodologias utilizadas nos estudos realizados?

Quais são as dimensões avaliadas no impacto da demência nos diferentes membros do agregado familiar? 


\section{METODOLOGIA}

A estratégia scoping review consiste numa revisão sistematizada, exploratória, destinada a mapear na produção científica, estudos relevantes em determinada área. $O$ estudo seguiu os passos preconizados pelo Joanna Briggs Institute ${ }^{(6)}$ : formulação da questão de pesquisa, especificação dos métodos de seleção da literatura, detalhe do procedimento de extração de dados, avaliação dos resultados de acordo com a sua pertinência e validade e análise, extraindo os dados e sintetizando as conclusões.

Na scoping review a questão inicial é ampla; os critérios de inclusão e de exclusão podem ser estabelecidos à posteriori; a escolha dos estudos não foca na qualidade da investigação; pode ou não envolver extração de dados; a síntese é fundamentalmente qualitativa; é utilizada para identificar as variáveis e as lacunas existentes na literatura. Foram realizadas as seguintes etapas: identificação da questão principal; identificação de estudos mais relevantes; seleção dos estudos; metodologia utilizada; extração de dados; apresentação e discussão dos resultados. Com a revisão realizada procurou-se responder à questão de pesquisa, formulada e utilizando a estratégia de participantes, conceito e contexto (PCC).

Esta metodologia permite a obtenção de resultados mais abrangentes e com menor profundidade sobre uma temática, no entanto, como na metodologia da revisão sistemática, possui um desenho metódico, transparente e replicável. Existem algumas características que se diferenciam na revisão sistemática e na scoping review. De forma a responder à questão inicial foram utilizadas as seguintes palavras-chave: neurocognitive disorder, family relations, nursing, adaptation e os respetivos termos em Português e Espanhol. A pesquisa foi realizada no período de janeiro de 2012 a dezembro de 2018. Adotou-se a estratégia de pesquisa das 3 etapas ${ }^{(6)}$, sendo que a primeira se iniciou com uma pesquisa inicial nas bases de dados EBSCO-host, Web of Science, B-on e PubMed que permitiu a identificação das palavras utilizadas nos títulos e resumos, bem como os termos de indexação. Posteriormente, numa segunda etapa, realizou-se a pesquisa nas referidas bases de dados e na ferramenta Google Scholar.

Na base de dados PubMed foram utilizadas as seguintes combinações: (("neurocognitive disorders"[MeSH Terms] OR ("neurocognitive"[All Fields] AND "disorders"[All Fields]) OR "neurocognitive disorders"[All Fields] OR ("neurocognitive"[All Fields] AND "disorder"[All Fields]) OR "neurocognitive disorder"[All Fields]) AND ("family relations"[MeSH Terms] OR ("family"[All Fields] AND "relations"[All Fields]) OR "family relations"[All Fields]) AND ("nursing"[Subheading] OR "nursing"[All Fields] OR "nursing"[MeSH Terms] OR "nursing"[All Fields] OR "adaptation"[All Fields])) AND ("free full text"[sb] AND "2012/10/05"[PDat] : "2018/09/03"[PDat]. Uma vez que a scoping review é exploratória, os resultados encontrados acerca do tema devem ser incluídos, permitindo que os pesquisadores identifiquem lacunas na investigação existente ${ }^{(7)}$. A terceira etapa consistiu na pesquisa de listas de referências da literatura relevante para encontrar estudos adicionais. Os critérios de inclusão foram os artigos publicados entre 2012 e 2018. A pesquisa de artigos foi realizada por dois pesquisadores separadamente, com base nas informações fornecidas no título e resumo, em idioma português, inglês e espanhol e disponíveis em free full text.

Foram excluídos os artigos relativos a outros diagnósticos e estudos com desenho de pesquisa ou objetivos pouco definidos. Para a análise dos resultados, foi criada uma tabela de evidências, que permitiu a análise de acordo com os seguintes itens: base de dados, ano, autores, nome do artigo, objetivos, metodologia utilizada, indicadores estudados e principais resultados.

\section{RESULTADOS}

Foram encontrados 7 artigos na base PubMed, 12 artigos na b-on, 8 na EBSCO-host tendo sido incluídos 27 artigos, de acordo com os critérios definidos. Numa terceira fase, as referências 
bibliográficas de todos os artigos identificados foram analisadas para identificar outros estudos relevantes tendo sido incluídos 2 estudos. Na figura 1, apresentamos o percurso da seleção dos estudos identificados.

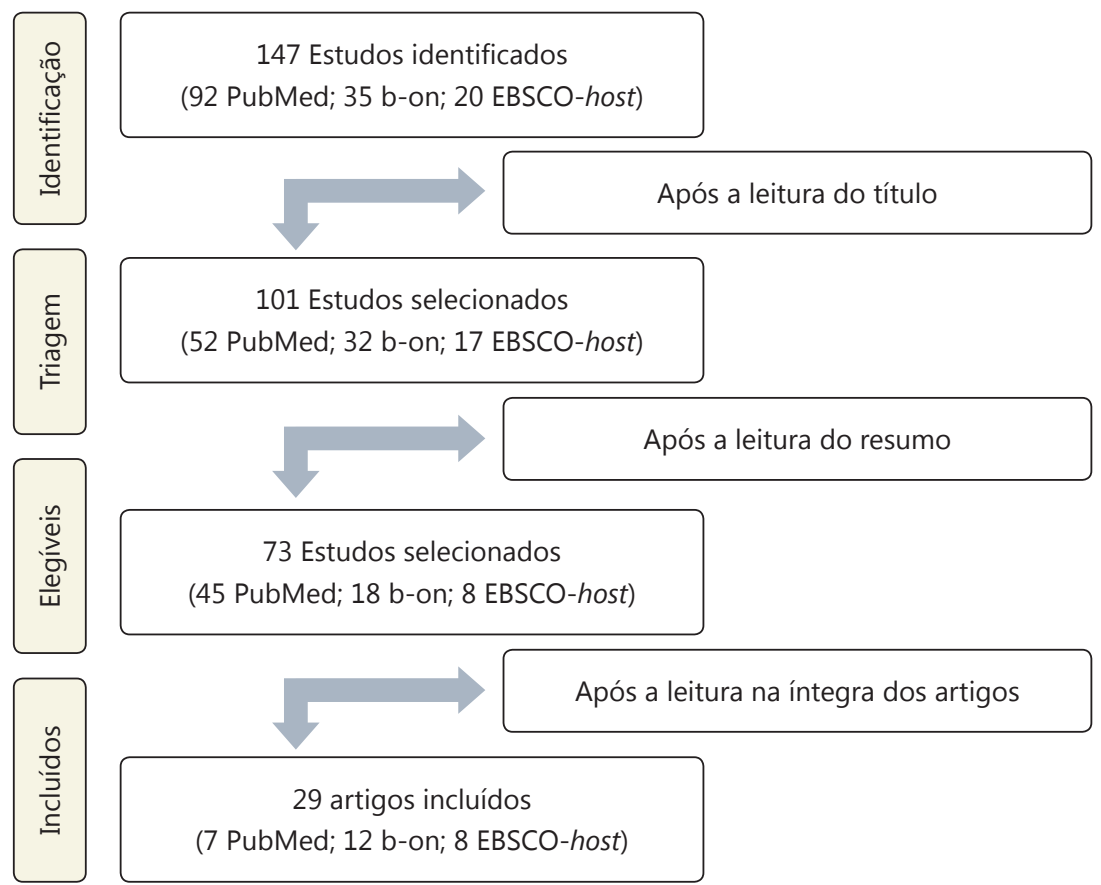

Figura 1 - Fluxograma PRISMA (adaptado) do processo de seleção dos estudos.

Quadro 1 - Artigos Incluídos na Revisão Scoping da Literatura.

\begin{tabular}{|c|c|c|c|c|c|c|}
\hline $\begin{array}{l}\text { Ano/Autor } \\
\text { Principal }\end{array}$ & Estudos & Objetivos & Metodologia & $\begin{array}{c}\text { População } \\
\text { alvo }\end{array}$ & Indicadores & Principais resultados \\
\hline $\begin{array}{l}2018^{\star \star \star *} \\
\text { Fernandes, et } \\
\text { al. }{ }^{(8)}\end{array}$ & $\begin{array}{l}\text { Family caregivers of } \\
\text { dependent elderly: } \\
\text { same needs, } \\
\text { different contexts } \\
\text { - a focus group } \\
\text { analysis }\end{array}$ & $\begin{array}{l}\text { Identificar as } \\
\text { necessidades do } \\
\text { cuidador familiar do } \\
\text { idoso dependente. }\end{array}$ & $\begin{array}{l}\text { Fenomenológico, } \\
\text { análise da } \\
\text { narrativa de } 12 \\
\text { cuidadores }\end{array}$ & $\begin{array}{l}\text { Cuidadores } \\
\text { de familiares } \\
\text { dependentes } \\
\text { em Portugal } \\
\text { e Brasil }\end{array}$ & $\begin{array}{l}\text { Necessidades } \\
\text { identificadas }\end{array}$ & $\begin{array}{l}\text { Categorias emergentes: } \\
\text { a necessidade de } \\
\text { aprender sozinho; as } \\
\text { necessidades de tempo } \\
\text { para a manutenção de } \\
\text { papeis; a necessidade } \\
\text { de uma rede de } \\
\text { suporte, a resiliência } \\
\text { nos desequilíbrios } \\
\text { familiares e os recursos } \\
\text { para o cuidar. }\end{array}$ \\
\hline $\begin{array}{l}2018^{* * * *} \\
\text { Correia, et al. }{ }^{(9)}\end{array}$ & $\begin{array}{l}\text { CUIDAOAZ: } \\
\text { satisfação dos } \\
\text { cuidadores } \\
\text { familiares de idosos } \\
\text { dependentes em } \\
\text { Oliveira de Azeméis. }\end{array}$ & $\begin{array}{l}\text { Caracterizar os } \\
\text { motivos que } \\
\text { conduzem à } \\
\text { assunção do papel } \\
\text { de cuidador familiar; } \\
\text { Determinar a } \\
\text { satisfação com o } \\
\text { exercício do papel } \\
\text { e as principais } \\
\text { fontes de satisfação; } \\
\text { determinar a } \\
\text { perceção de } \\
\text { autoeficácia para } \\
\text { tomar conta e o } \\
\text { envolvimento nos } \\
\text { cuidados. }\end{array}$ & $\begin{array}{l}\text { Mista, descritiva } \\
\text { e transversal }\end{array}$ & $\begin{array}{l}\text { Cuidadores } \\
\text { familiares } \\
\text { de idosos } \\
\text { dependentes }\end{array}$ & $\begin{array}{l}\text { Satisfação do } \\
\text { cuidador informal }\end{array}$ & $\begin{array}{l}\text { Satisfação elevada na } \\
\text { assunção do papel; } \\
\text { principais fontes de } \\
\text { satisfação relacionam- } \\
\text { se com a pessoa } \\
\text { dependente como } \\
\text { principal beneficiária. } \\
\text { Principais dificuldades } \\
\text { no desempenho da } \\
\text { prestação de cuidados } \\
\text { e gestão de emoções. }\end{array}$ \\
\hline
\end{tabular}


Continuação do Quadro 1

\begin{tabular}{|c|c|c|c|c|c|c|}
\hline $\begin{array}{l}\text { Ano/Autor } \\
\text { Principal }\end{array}$ & Estudos & Objetivos & Metodologia & $\begin{array}{c}\text { População } \\
\text { alvo }\end{array}$ & Indicadores & Principais resultados \\
\hline $\begin{array}{l}2017^{*} \\
\text { Oliveira et } \\
\text { al. }{ }^{(10)}\end{array}$ & $\begin{array}{l}\text { Impacto da doença } \\
\text { de Alzheimer para } \\
\text { o familiar cuidador: } \\
\text { Uma revisão } \\
\text { integrativa }\end{array}$ & $\begin{array}{l}\text { Analisar o impacto } \\
\text { da doença na vida } \\
\text { do cuidador familiar. }\end{array}$ & $\begin{array}{l}\text { Revisão } \\
\text { integrativa }\end{array}$ & Cuidador & $\begin{array}{l}\text { Sobrecarga física } \\
\text { e emocional; } \\
\text { Conhecimento }\end{array}$ & $\begin{array}{l}\text { Aumento da } \\
\text { sobrecarga física, } \\
\text { emocional; falta de } \\
\text { conhecimentos sobre a } \\
\text { doença e como agir. }\end{array}$ \\
\hline $\begin{array}{l}2017^{\star} \\
\text { Farina, } \\
\text { et al. }{ }^{(11)}\end{array}$ & $\begin{array}{l}\text { Factors associated } \\
\text { with the quality } \\
\text { of life of family } \\
\text { carers of people } \\
\text { with dementia: A } \\
\text { systematic review }\end{array}$ & $\begin{array}{l}\text { Identificar fatores } \\
\text { relacionados à } \\
\text { Qualidade de Vida } \\
\text { de cuidadores } \\
\text { familiares. }\end{array}$ & $\begin{array}{l}\text { Revisão } \\
\text { sistemática } \\
41 \text { estudos }\end{array}$ & Cuidador & Qualidade de Vida & $\begin{array}{l}\text { A qualidade de } \\
\text { vida é afetada pela: } \\
\text { demografia; relação } \\
\text { cuidador-paciente; } \\
\text { características de } \\
\text { demência; saúde e } \\
\text { bem-estar emocional; } \\
\text { suporte recebido; } \\
\text { independência; auto- } \\
\text { eficácia e futuro. }\end{array}$ \\
\hline $\begin{array}{l}2017^{\star \star} \\
\text { Chen, } \\
\text { et al. }{ }^{(12)}\end{array}$ & $\begin{array}{l}\text { The Relationship } \\
\text { between "What We } \\
\text { Believe" and "How } \\
\text { We Care" Among } \\
\text { Daughters Caring } \\
\text { for a Parent with } \\
\text { Dementia }\end{array}$ & $\begin{array}{l}\text { Entender os fatores } \\
\text { associados ao } \\
\text { conflito entre as } \\
\text { filhas e pais com } \\
\text { demência. }\end{array}$ & $\begin{array}{l}77 \text { filhas } \\
\text { cuidadoras } \\
\text { primárias de um } \\
\text { dos pais com } \\
\text { demência. }\end{array}$ & Cuidador & $\begin{array}{l}\text { Conflitos } \\
\text { relacionais }\end{array}$ & $\begin{array}{l}\text { Maior intensidade da } \\
\text { crença das filhas de } \\
\text { que seus pais podem } \\
\text { controlar sintomas } \\
\text { relacionados à } \\
\text { demência foi associada } \\
\text { a mais conflitos de } \\
\text { relacionamento. }\end{array}$ \\
\hline $\begin{array}{l}2017^{\star \star} \\
\text { Bjorge, et al. }{ }^{(13)}\end{array}$ & $\begin{array}{l}\text { Relationship Quality } \\
\text { and Distress in } \\
\text { Caregivers of } \\
\text { Persons with } \\
\text { Dementia: A Cross- } \\
\text { Sectional Study. }\end{array}$ & $\begin{array}{l}\text { Investigar a relação } \\
\text { entre cuidadores } \\
\text { e recetores de } \\
\text { cuidados, definidos } \\
\text { como membros da } \\
\text { família domiciliária } \\
\text { com demência }\end{array}$ & $\begin{array}{l}\text { Incluídos } \\
208 \text { pares de } \\
\text { cuidadores/ } \\
\text { cuidado }\end{array}$ & $\begin{array}{l}\text { Díade } \\
\text { cuidador/ } \\
\text { pessoa } \\
\text { cuidada }\end{array}$ & $\begin{array}{l}\text { Dificuldade em } \\
\text { cuidar e tempo } \\
\text { dispensado }\end{array}$ & $\begin{array}{l}\text { As perceções dos } \\
\text { cuidadores foram: } \\
\text { dificuldade no cuidar } \\
\text { e a quantidade de } \\
\text { tempo gasto. }\end{array}$ \\
\hline $\begin{array}{l}2017^{*} \\
\text { Weisman, et } \\
\text { al. }{ }^{(14)}\end{array}$ & $\begin{array}{l}\text { Free will } \\
\text { perceptions, } \\
\text { religious coping, } \\
\text { and other mental } \\
\text { health outcomes } \\
\text { in caregivers of } \\
\text { individuals with } \\
\text { dementia }\end{array}$ & $\begin{array}{l}\text { Testar as hipóteses } \\
\text { maiores perceções } \\
\text { de livre arbítrio } \\
\text { e enfrentamento } \\
\text { religioso estarão } \\
\text { associados a uma } \\
\text { maior qualidade } \\
\text { de vida e outros } \\
\text { indicadores de saúde } \\
\text { mental. }\end{array}$ & $\begin{array}{l}\text { Amostra de } 107 \\
\text { pacientes com } \\
\text { demência }\end{array}$ & Pacientes & Qualidade de Vida & $\begin{array}{l}\text { As perceções de } \\
\text { vontade livre, o } \\
\text { enfrentamento } \\
\text { religioso, os } \\
\text { relacionamentos, estão } \\
\text { associados a uma } \\
\text { maior qualidade de } \\
\text { vida. } \\
\text { Indicadores que } \\
\text { surgiram ansiedade e } \\
\text { depressão. }\end{array}$ \\
\hline $\begin{array}{l}2017^{\star} \\
\text { Cesário, et } \\
\text { al. }{ }^{(15)}\end{array}$ & $\begin{array}{l}\text { Estresse e } \\
\text { qualidade de } \\
\text { vida do cuidador } \\
\text { familiar de idoso } \\
\text { portador da doença } \\
\text { de Alzheimer }\end{array}$ & $\begin{array}{l}\text { Analisar a relação } \\
\text { entre o estresse e a } \\
\text { qualidade de vida do } \\
\text { cuidador familiar de } \\
\text { idosos portadores da } \\
\text { doença de Alzheimer }\end{array}$ & $\begin{array}{l}\text { Estudo } \\
\text { quantitativo } \\
\text { descritivo, } \\
\text { entrevistas } \\
\text { semiestruturadas } \\
\text { a } 43 \text { cuidadores }\end{array}$ & Cuidadores & Qualidade de Vida & $\begin{array}{l}\text { Os cuidadores } \\
\text { apresentam fracas } \\
\text { condições de saúde } \\
\text { a nível de stress } \\
\text { e diminuição da } \\
\text { qualidade de vida. }\end{array}$ \\
\hline $\begin{array}{l}2017^{* \star *} \\
\text { Marins, } \\
\text { et al. }\end{array}$ & $\begin{array}{l}\text { O impacto do } \\
\text { comportamento do } \\
\text { idoso com doença } \\
\text { de alzheimer na } \\
\text { vida do cuidador }\end{array}$ & $\begin{array}{l}\text { Refletir sobre o } \\
\text { comportamento do } \\
\text { idoso com doença } \\
\text { de Alzheimer e suas } \\
\text { implicações na vida } \\
\text { do cuidador. }\end{array}$ & $\begin{array}{l}\text { Reflexão teórica } \\
\text { fundamentada } \\
\text { nos preceitos do } \\
\text { Interacionismo } \\
\text { Simbólico. }\end{array}$ & Cuidador & Comportamento & $\begin{array}{l}\text { À medida que vivencia } \\
\text { a realidade de cuidado, } \\
\text { são estabelecidas } \\
\text { interações que se } \\
\text { desdobram em } \\
\text { demandas de cuidado. }\end{array}$ \\
\hline
\end{tabular}




\begin{tabular}{|c|c|c|c|c|c|c|}
\hline $\begin{array}{l}\text { Ano/Autor } \\
\text { Principal }\end{array}$ & Estudos & Objetivos & Metodologia & $\begin{array}{c}\text { População } \\
\text { alvo }\end{array}$ & Indicadores & Principais resultados \\
\hline $\begin{array}{l}2017^{*} \\
\text { Baggio, } \\
\text { et al. }{ }^{(17)}\end{array}$ & $\begin{array}{l}\text { O Impacto da } \\
\text { doença de } \\
\text { Alzheimer no } \\
\text { cuidador: reflexões } \\
\text { sobre degeneração } \\
\text { e desequilíbrio } \\
\text { familiar }\end{array}$ & $\begin{array}{l}\text { Identificar os } \\
\text { principais impactos } \\
\text { sofridos na vida do } \\
\text { cuidador familiar e } \\
\text { restantes membros } \\
\text { da família. }\end{array}$ & $\begin{array}{l}\text { Estudo } \\
\text { qualitativo } \\
\text { descritivo com } \\
\text { entrevistas a } \\
\text { cinco cuidadores } \\
\text { familiares }\end{array}$ & Cuidador & $\begin{array}{l}\text { Conhecimento; } \\
\text { Sobrecarga física } \\
\text { e emocional }\end{array}$ & $\begin{array}{l}\text { Falta de conhecimento } \\
\text { em relação à doença; } \\
\text { o ato de cuidar mostra } \\
\text { elevados níveis de } \\
\text { sobrecarga física } \\
\text { e emocional bem } \\
\text { como medo, stress, } \\
\text { ansiedade, angústia e } \\
\text { preocupação. }\end{array}$ \\
\hline $\begin{array}{l}2017^{*} \\
\text { Leite, } \\
\text { et al. }{ }^{(18)}\end{array}$ & $\begin{array}{l}\text { A vulnerabilidade } \\
\text { dos cuidadores } \\
\text { de idosos com } \\
\text { demência: revisão } \\
\text { integrativa }\end{array}$ & $\begin{array}{l}\text { Analisar a } \\
\text { vulnerabilidade dos } \\
\text { cuidadores de idosos } \\
\text { com demência. }\end{array}$ & $\begin{array}{l}\text { Revisão } \\
\text { Integrativa de } \\
\text { natureza quanti- } \\
\text { qualitativa }\end{array}$ & Cuidador & $\begin{array}{l}\text { Sobrecarga } \\
\text { emocional e física }\end{array}$ & $\begin{array}{l}\text { Aumento da } \\
\text { sobrecarga emocional, } \\
\text { stress diário e } \\
\text { sintomas depressivos. } \\
\text { Medidas atenuantes } \\
\text { para aliviar o stresse } \\
\text { diário, a prática } \\
\text { diária de meditação } \\
\text { yoga, a religiosidade, } \\
\text { o relacionamento } \\
\text { positivo entre a família, } \\
\text { o idoso e o cuidador. }\end{array}$ \\
\hline $\begin{array}{l}2016^{\star} \\
\text { Folle, } \\
\text { et al. }{ }^{(19)}\end{array}$ & $\begin{array}{l}\text { Social } \\
\text { Representation } \\
\text { of Alzheimer's } \\
\text { disease for family } \\
\text { caregivers: stressful } \\
\text { and rewarding }\end{array}$ & $\begin{array}{l}\text { Compreender } \\
\text { o conteúdo da } \\
\text { representação } \\
\text { social de cuidadores } \\
\text { familiares de } \\
\text { pacientes dom } \\
\text { Alzheimer }\end{array}$ & $\begin{array}{l}\text { Entrevistas com } \\
26 \text { cuidadores. }\end{array}$ & Cuidador & $\begin{array}{l}\text { Sobrecarga } \\
\text { emocional, física; } \\
\text { Isolamento social }\end{array}$ & $\begin{array}{l}\text { Os cuidadores privam- } \\
\text { se de viver as suas } \\
\text { próprias vidas para } \\
\text { dedicar-se a cuidar } \\
\text { levando a desgaste } \\
\text { físico, emocional e } \\
\text { isolamento social. }\end{array}$ \\
\hline $\begin{array}{l}2016^{\star \star \star} \\
\text { Zauszniewski, } \\
\text { et al. } \\
.(20)\end{array}$ & $\begin{array}{l}\text { Resourcefulness } \\
\text { Training for } \\
\text { Dementia } \\
\text { Caregivers: } \\
\text { Establishing Fidelity. }\end{array}$ & $\begin{array}{l}\text { Testar a eficácia do } \\
\text { registo no diário } \\
\text { e da gravação de } \\
\text { voz como métodos } \\
\text { de treinamento de } \\
\text { treinamento de } \\
\text { recursos (RT) }\end{array}$ & $\begin{array}{l}\text { Teste piloto com } \\
63 \text { cuidadores de } \\
\text { mulheres }\end{array}$ & Cuidador & $\begin{array}{l}\text { Treino de } \\
\text { habilidades }\end{array}$ & $\begin{array}{l}\text { A fidelidade de } \\
\text { implementação da RT } \\
\text { permite escolher um } \\
\text { método para reforçar } \\
\text { suas habilidades em } \\
\text { recursos humanos. }\end{array}$ \\
\hline $\begin{array}{l}2016^{\star \star} \\
\text { Manzini, et } \\
a l^{(21)}\end{array}$ & $\begin{array}{l}\text { Fatores associados } \\
\text { à resiliência de } \\
\text { cuidador familiar } \\
\text { de pessoa com } \\
\text { demência: revisão } \\
\text { sistemática }\end{array}$ & $\begin{array}{l}\text { Identificar fatores } \\
\text { associados ao } \\
\text { desenvolvimento } \\
\text { da resiliência em } \\
\text { cuidador familiar } \\
\text { de pessoa com } \\
\text { demência e os } \\
\text { desfechos da } \\
\text { resiliência para o } \\
\text { cuidador e o recetor } \\
\text { de cuidados. }\end{array}$ & $\begin{array}{l}\text { Revisão } \\
\text { sistemática }\end{array}$ & Cuidador & $\begin{array}{l}\text { Fatores associados } \\
\text { ao cuidar }\end{array}$ & $\begin{array}{l}\text { Os fatores associados: } \\
\text { depressão, ansiedade, } \\
\text { sobrecarga, uso de } \\
\text { medicamentos, grau } \\
\text { de parentesco com o } \\
\text { recetor de cuidados e } \\
\text { condições de saúde. }\end{array}$ \\
\hline $\begin{array}{l}2016^{\star *} \\
\text { Kupeli, et al. (22) }\end{array}$ & $\begin{array}{l}\text { Context, } \\
\text { mechanisms and } \\
\text { outcomes in end of } \\
\text { life care for people } \\
\text { with advanced } \\
\text { dementia }\end{array}$ & $\begin{array}{l}\text { Explorar o contexto, } \\
\text { mecanismos e } \\
\text { resultados para } \\
\text { proporcionar bons } \\
\text { cuidados paliativos. }\end{array}$ & $\begin{array}{l}\text { Estudo } \\
\text { qualitativo com } \\
14 \text { profissionais } \\
\text { de saúde com } \\
\text { a realização } \\
\text { de entrevistas } \\
\text { interativas em } \\
\text { residências do } \\
\text { Reino Unido. }\end{array}$ & $\begin{array}{l}\text { Profissionais } \\
\text { de saúde }\end{array}$ & $\begin{array}{l}\text { Resultados } \\
\text { dos cuidados } \\
\text { paliativos } \\
\text { prestados }\end{array}$ & $\begin{array}{l}\text { O bom atendimento } \\
\text { paliativo deve-se } \\
\text { a priorização de } \\
\text { cuidados psicossociais } \\
\text { e espirituais, } \\
\text { relacionamentos } \\
\text { com cuidadores, } \\
\text { atendimento de } \\
\text { necessidades físicas, } \\
\text { gestão de sintomas e } \\
\text { cuidados integrados } \\
\text { por uma equipe } \\
\text { multidisciplinar. }\end{array}$ \\
\hline
\end{tabular}


Continuação do Quadro 1

\begin{tabular}{|c|c|c|c|c|c|c|}
\hline $\begin{array}{l}\text { Ano/Autor } \\
\text { Principal }\end{array}$ & Estudos & Objetivos & Metodologia & $\begin{array}{c}\text { População } \\
\text { alvo }\end{array}$ & Indicadores & Principais resultados \\
\hline $\begin{array}{l}2015^{\star \star} \\
\text { Chiao et al. }\end{array}$ & $\begin{array}{l}\text { Caregiver burden } \\
\text { for informal } \\
\text { caregivers of } \\
\text { patients with } \\
\text { dementia: A } \\
\text { systematic review }\end{array}$ & $\begin{array}{l}\text { Identificar os fatores } \\
\text { que constituem a } \\
\text { carga do cuidador } \\
\text { sobre cuidadores } \\
\text { informais de pessoas } \\
\text { com demência } \\
\text { que vivem na } \\
\text { comunidade. }\end{array}$ & $\begin{array}{l}\text { Revisão } \\
\text { sistemática }\end{array}$ & Cuidadores & $\begin{array}{l}\text { Fatores associados } \\
\text { ao cuidar }\end{array}$ & $\begin{array}{l}\text { Problemas } \\
\text { comportamentais, } \\
\text { fatores sócio- } \\
\text { demográficos } \\
\text { e psicológicos } \\
\text { estão associados } \\
\text { à sobrecarga do } \\
\text { cuidador. }\end{array}$ \\
\hline $\begin{array}{l}2015^{\star *} \\
\text { Alvira, } \\
\text { et al. }\end{array}$ & $\begin{array}{l}\text { The association } \\
\text { between positive- } \\
\text { negative reactions } \\
\text { of informal } \\
\text { caregivers of } \\
\text { people with } \\
\text { dementia and } \\
\text { health outcomes } \\
\text { in eight European } \\
\text { countries: a cross- } \\
\text { sectional study. }\end{array}$ & $\begin{array}{l}\text { Descrever as } \\
\text { associações entre } \\
\text { reações positivas } \\
\text { e negativas de } \\
\text { cuidadores informais } \\
\text { de pessoas com } \\
\text { demência. }\end{array}$ & $\begin{array}{l}\text { Estudo de } \\
\text { associação } \\
\text { baseado } \\
\text { em dados } \\
\text { transversais } \\
\text { em oito países } \\
\text { europeus. }\end{array}$ & Cuidador & $\begin{array}{l}\text { Sobrecarga } \\
\text { Bem-estar } \\
\text { psicológico } \\
\text { Qualidade de vida }\end{array}$ & $\begin{array}{l}\text { A auto-estima e a } \\
\text { falta de apoio familiar } \\
\text { correlacionaram-se } \\
\text { com a sobrecarga, } \\
\text { bem-estar psicológico } \\
\text { e qualidade de vida. }\end{array}$ \\
\hline $\begin{array}{l}2015^{\star \star \star} \\
\text { Gilson, } \\
\text { et al. }\end{array}$ & $\begin{array}{l}\text { Os impactos na } \\
\text { saúde do cuidador } \\
\text { familiar de pessoas } \\
\text { com doença de } \\
\text { alzheimer: uma } \\
\text { revisão bibliográfica }\end{array}$ & $\begin{array}{l}\text { Identificar os } \\
\text { impactos na } \\
\text { saúde do cuidador } \\
\text { familiar de pessoas } \\
\text { com Doença de } \\
\text { Alzheimer }\end{array}$ & $\begin{array}{l}\text { Revisão narrativa } \\
\text { da literatura }\end{array}$ & Cuidador & $\begin{array}{l}\text { Sobrecarga física } \\
\text { e emocional } \\
\text { Qualidade de vida }\end{array}$ & $\begin{array}{l}\text { Com o avanço da } \\
\text { doença, o desgaste } \\
\text { físico e emocional } \\
\text { passa a afetar a } \\
\text { qualidade de vida. } \\
\text { O cuidador dedica o } \\
\text { seu tempo e energia, } \\
\text { denotando-se anseios } \\
\text { e dificuldades pessoais. }\end{array}$ \\
\hline $\begin{array}{l}2014^{*} \\
\text { Matsushita, et } \\
a^{(26)}\end{array}$ & $\begin{array}{l}\text { Is sense of } \\
\text { coherence helpful } \\
\text { in coping with } \\
\text { caregiver burden } \\
\text { for dementia? }\end{array}$ & $\begin{array}{l}\text { Examinar a relação } \\
\text { entre sobrecarga e } \\
\text { sentido da coerência } \\
\text { (SOC) entre } \\
\text { cuidadores }\end{array}$ & $\begin{array}{l}274 \text { Entrevistas } \\
\text { semi- } \\
\text { estruturadas }\end{array}$ & $\begin{array}{l}\text { Cuidador ou } \\
\text { membro da } \\
\text { família }\end{array}$ & Sobrecarga & $\begin{array}{l}\text { Entre } 78 \\
\text { cuidadores com } \\
\text { comprometimento } \\
\text { cognitivo, o score } \\
\text { Zarit Caregiver Burden } \\
\text { Interview (ZBI) foi } \\
\text { significativamente } \\
\text { associado com SOC } \\
\text { estando associada } \\
\text { à tensão pessoal no } \\
\text { ZBI. O reforço do SOC } \\
\text { contribui para reduzir a } \\
\text { tensão pessoal. }\end{array}$ \\
\hline $\begin{array}{l}2014^{*} \\
\text { Gibson, et } \\
\text { al. }^{(27)}\end{array}$ & $\begin{array}{l}\text { Understanding the } \\
\text { sleep problems } \\
\text { of people with } \\
\text { dementia and their } \\
\text { family caregivers }\end{array}$ & $\begin{array}{l}\text { Compreender os } \\
\text { diferentes tipos de } \\
\text { problemas de sono } \\
\text { vivenciados por } \\
\text { casais que vivem na } \\
\text { comunidade; } \\
\text { Identificar as } \\
\text { estratégias para gerir } \\
\text { o sono }\end{array}$ & $\begin{array}{l}12 \text { díades } \\
\text { cuidador/ } \\
\text { cuidado de } \\
\text { serviço de apoio } \\
\text { domicílio na } \\
\text { Nova Zelândia }\end{array}$ & $\begin{array}{l}\text { Díade } \\
\text { cuidador/ } \\
\text { pessoa } \\
\text { cuidada }\end{array}$ & $\begin{array}{l}\text { Alterações padrão } \\
\text { do sono }\end{array}$ & $\begin{array}{l}\text { Os distúrbios variam } \\
\text { desde problemas de } \\
\text { sono relacionados à } \\
\text { idade exacerbados } \\
\text { até períodos } \\
\text { irregulares de sono } \\
\text { e comportamentos } \\
\text { disruptivos à noite. }\end{array}$ \\
\hline $\begin{array}{l}2014^{*} \\
\text { Crellin, et al. }{ }^{(28)}\end{array}$ & $\begin{array}{l}\text { Self-efficacy and } \\
\text { health-related } \\
\text { quality of life } \\
\text { in family carers } \\
\text { of people with } \\
\text { dementia: a } \\
\text { systematic review. }\end{array}$ & $\begin{array}{l}\text { Explorar o papel } \\
\text { da auto-eficácia na } \\
\text { qualidade de vida } \\
\text { relacionada com a } \\
\text { saúde dos familiares } \\
\text { de pessoas com } \\
\text { demência }\end{array}$ & $\begin{array}{l}17 \text { estudos } \\
\text { quantitativos, } 4 \\
\text { qualitativos e } 1 \\
\text { método misto. } \\
\text { Construído um } \\
\text { modelo que } \\
\text { descreve o } \\
\text { papel da tarefas } \\
\text { específicas, } \\
\text { crenças em } \\
\text { saúde }\end{array}$ & Cuidador & Qualidade de vida & $\begin{array}{l}\text { Maior exploração das } \\
\text { crenças e da influência } \\
\text { das caraterísticas do } \\
\text { cuidador. }\end{array}$ \\
\hline
\end{tabular}




\begin{tabular}{|c|c|c|c|c|c|c|}
\hline $\begin{array}{c}\text { Ano/Autor } \\
\text { Principal }\end{array}$ & Estudos & Objetivos & Metodologia & $\begin{array}{c}\text { População } \\
\text { alvo }\end{array}$ & Indicadores & Principais resultados \\
\hline $\begin{array}{l}2014^{* * *} \\
\text { Seima, } \\
\text { et al. }\end{array}$ & $\begin{array}{l}\text { Relação no cuidado } \\
\text { entre o cuidador } \\
\text { familiar e o idoso } \\
\text { com alzheimer }\end{array}$ & $\begin{array}{l}\text { Interpretar a relação } \\
\text { no cuidado entre } \\
\text { cuidadores familiares } \\
\text { e idosos com } \\
\text { Alzheimer, alicerçada } \\
\text { na dialética concreta } \\
\text { da participação } \\
\text { segundo os quatro } \\
\text { preceitos de Gabriel } \\
\text { Marcel }\end{array}$ & $\begin{array}{l}\text { Estudo } \\
\text { quantitativo, de } \\
\text { corte transversal, } \\
\text { e qualitativo- } \\
\text { descritivo. } \\
208 \text { cuidadores } \\
\text { na etapa } \\
\text { quantitativa e } 36 \\
\text { na qualitativa. }\end{array}$ & Cuidador & $\begin{array}{l}\text { Relações } \\
\text { familiares entre } \\
\text { cuidador e } \\
\text { paciente }\end{array}$ & $\begin{array}{l}\text { A relação envolve } \\
\text { amor, felicidade, } \\
\text { esperança e presença. } \\
\text { O enfermeiro } \\
\text { desenvolver a } \\
\text { sensibilidade e } \\
\text { a criatividade } \\
\text { considerando a } \\
\text { subjetividade e o } \\
\text { mistério das relações } \\
\text { humanas. }\end{array}$ \\
\hline $\begin{array}{l}2014^{\star * *} \\
\text { Bremen- } \\
\text { Kamp, } \\
\text { et al. }\end{array}$ & $\begin{array}{l}\text { Sintomas } \\
\text { neuropsiquiátricos } \\
\text { na doença de } \\
\text { Alzheimer: } \\
\text { frequência, } \\
\text { correlação e } \\
\text { ansiedade do } \\
\text { cuidador. }\end{array}$ & $\begin{array}{l}\text { Identificar sintomas } \\
\text { neuropsiquiátricos } \\
\text { mais frequentes e de } \\
\text { maior gravidade nos } \\
\text { pacientes portadores } \\
\text { da doença de } \\
\text { Alzheimer; } \\
\text { Especificar quais } \\
\text { os distúrbios mais } \\
\text { desgastantes pelo } \\
\text { cuidador; } \\
\text { Correlacionar } \\
\text { os sintomas } \\
\text { neuropsiquiátricos }\end{array}$ & $\begin{array}{l}\text { Estudo } \\
\text { transversal, } \\
\text { descritivo } \\
\text { exploratório com } \\
50 \text { idosos com } \\
\text { provável doença } \\
\text { de Alzheimer, em } \\
\text { ambulatório de } \\
\text { uma instituição } \\
\text { filantrópica } \\
\text { de Vitória, } \\
\text { acompanhados } \\
\text { do cuidador. }\end{array}$ & Pacientes & $\begin{array}{l}\text { Lidar com } \\
\text { os sintomas } \\
\text { neuropsiquiátricos }\end{array}$ & $\begin{array}{l}\text { Reforçam a } \\
\text { necessidade de } \\
\text { estudos sobre a } \\
\text { abordagem das } \\
\text { manifestações } \\
\text { neuropsiquiátricas, } \\
\text { dada sua alta } \\
\text { prevalência e à } \\
\text { presença de sintomas } \\
\text { concomitantes que } \\
\text { geram alto grau de } \\
\text { desgaste no cuidador. }\end{array}$ \\
\hline $\begin{array}{l}2014^{*} \\
\text { Llanque, } \\
\text { et al. }{ }^{(31)}\end{array}$ & $\begin{array}{l}\text { Concept Analysis: } \\
\text { Alzheimer's } \\
\text { Caregiver Stress }\end{array}$ & $\begin{array}{l}\text { Analisar o conceito } \\
\text { de estresse do } \\
\text { cuidador no } \\
\text { contexto de cuidar } \\
\text { de uma pessoa com } \\
\text { doença de Alzheimer }\end{array}$ & $\begin{array}{l}\text { Revisão da } \\
\text { literatura }\end{array}$ & Cuidador & $\begin{array}{l}\text { Sobrecarga do } \\
\text { cuidador }\end{array}$ & $\begin{array}{l}\text { O stress do cuidador } \\
\text { é a troca desigual } \\
\text { de assistência entre } \\
\text { pessoas que se } \\
\text { relacionam resultando } \\
\text { em stress emocional } \\
\text { e físico. }\end{array}$ \\
\hline $\begin{array}{l}2013^{\star \star} \\
\text { Neumann, et } \\
\text { al. }^{(32)}\end{array}$ & $\begin{array}{l}\text { Alzheimer's disease: } \\
\text { what changes in the } \\
\text { lifestyle of family } \\
\text { caregiver? }\end{array}$ & $\begin{array}{l}\text { Identificar as } \\
\text { mudanças } \\
\text { psicossociais que } \\
\text { ocorreram na } \\
\text { vida de familiares } \\
\text { cuidadores de idosos } \\
\text { com a doença de } \\
\text { Alzheimer }\end{array}$ & $\begin{array}{l}\text { Estudo } \\
\text { qualitativo com } \\
\text { entrevista semi- } \\
\text { estruturada a } 6 \\
\text { cuidadores num } \\
\text { hospital público. }\end{array}$ & Cuidador & $\begin{array}{l}\text { Projetos de vida } \\
\text { adiados }\end{array}$ & $\begin{array}{l}\text { Áreas da vida afetadas: } \\
\text { familiar, social, saúde } \\
\text { mental e física, } \\
\text { exigindo adiar os } \\
\text { projetos de vida. }\end{array}$ \\
\hline $\begin{array}{l}2013^{\star * *} \\
\text { Rocha, } \\
\text { et al. }\end{array}$ & $\begin{array}{l}\text { Idoso em situação } \\
\text { de dependência: } \\
\text { estresse e coping } \\
\text { do cuidador } \\
\text { informal }\end{array}$ & $\begin{array}{l}\text { Estudar a relação } \\
\text { entre o estresse } \\
\text { psicológico (estresse) } \\
\text { e a adaptação } \\
\text { psicológica (coping) } \\
\text { do cuidador } \\
\text { informal do idoso } \\
\text { em situação de } \\
\text { dependência. }\end{array}$ & $\begin{array}{l}\text { Estudo } \\
\text { transversal } 110 \\
\text { cuidadores } \\
\text { informais no } \\
\text { domicílio do } \\
\text { Conselho de } \\
\text { Faro, Portugal. }\end{array}$ & Cuidador & $\begin{array}{l}\text { Estratégias de } \\
\text { coping }\end{array}$ & $\begin{array}{l}\text { As estratégias de } \\
\text { coping centradas no } \\
\text { problema e no meio } \\
\text { foram as utilizadas. As } \\
\text { estratégias centradas } \\
\text { no cuidador são as que } \\
\text { geram menor perceção } \\
\text { de stresse. }\end{array}$ \\
\hline $\begin{array}{l}2013^{\star \star \star} \\
\text { Andrade, } \\
\text { et al. }{ }^{(34)}\end{array}$ & $\begin{array}{l}\text { Reconfiguração } \\
\text { de campo do } \\
\text { familiar cuidador } \\
\text { do portador de } \\
\text { alzheimer }\end{array}$ & $\begin{array}{l}\text { Descrever a } \\
\text { reconfiguração do } \\
\text { familiar cuidador. }\end{array}$ & $\begin{array}{l}\text { Qualitativo, } \\
\text { perspetiva } \\
\text { fenomenológica } \\
\text { e na teoria de } \\
\text { campo com } 3 \\
\text { cuidadores. }\end{array}$ & Cuidador & $\begin{array}{l}\text { Reestruturação } \\
\text { familiar }\end{array}$ & $\begin{array}{l}\text { Quatro categorias } \\
\text { que descrevem a } \\
\text { reconfiguração: } \\
\text { assumindo a } \\
\text { responsabilidade em } \\
\text { cuidar; mudanças na } \\
\text { família; rede de apoio } \\
\text { social e estratégias de } \\
\text { enfrentamento. }\end{array}$ \\
\hline
\end{tabular}


Continuação do Quadro 1

\begin{tabular}{|c|c|c|c|c|c|c|}
\hline $\begin{array}{l}\text { Ano/Autor } \\
\text { Principal }\end{array}$ & Estudos & Objetivos & Metodologia & $\begin{array}{c}\text { População } \\
\text { alvo }\end{array}$ & Indicadores & Principais resultados \\
\hline $\begin{array}{l}2012^{\star \star \star *} \\
\text { Oliveira et } \\
\text { al. }{ }^{(35)}\end{array}$ & $\begin{array}{l}\text { As Repercussões } \\
\text { do Cuidado na } \\
\text { Vida do Cuidador } \\
\text { Familiar do Idoso } \\
\text { com Demência de } \\
\text { Alzheimer }\end{array}$ & $\begin{array}{l}\text { Investigar as } \\
\text { repercussões do } \\
\text { cuidado na vida do } \\
\text { cuidador familiar de } \\
\text { idoso com demência } \\
\text { de Alzheimer }\end{array}$ & $\begin{array}{l}\text { Qualitativo } 20 \\
\text { entrevistas a } \\
\text { cuidadores na } \\
\text { Associação } \\
\text { Brasileira de } \\
\text { Alzheimer do } \\
\text { estado do } \\
\text { Amazonas. }\end{array}$ & Cuidador & $\begin{array}{l}\text { Repercussão } \\
\text { social }\end{array}$ & $\begin{array}{l}\text { A privação social em } \\
\text { função do cuidado é } \\
\text { uma realidade. A raiva, } \\
\text { a impaciência, o medo, } \\
\text { a solidão e a vergonha } \\
\text { são sentimentos } \\
\text { vivenciados pelos } \\
\text { cuidadores que } \\
\text { se alternam com } \\
\text { sentimentos de } \\
\text { satisfação, felicidade, } \\
\text { compaixão e de } \\
\text { retribuição. }\end{array}$ \\
\hline $\begin{array}{l}2012^{*} \\
\text { Baptista et } \\
\text { al. }^{(36)}\end{array}$ & $\begin{array}{l}\text { A sobrecarga do } \\
\text { familiar cuidador no } \\
\text { âmbito domiciliar: } \\
\text { uma revisão } \\
\text { integrativa da } \\
\text { literatura. }\end{array}$ & $\begin{array}{l}\text { Identificar as } \\
\text { evidências acerca } \\
\text { dos fatores } \\
\text { geradores de } \\
\text { sobrecarga e sus } \\
\text { consequências } \\
\text { para os familiares } \\
\text { cuidadores de } \\
\text { adultos ou idosos }\end{array}$ & $\begin{array}{l}\text { Revisão } \\
\text { integrativa }\end{array}$ & Cuidador & $\begin{array}{l}\text { Sobrecarga do } \\
\text { cuidador }\end{array}$ & $\begin{array}{l}\text { Imposição do papel de } \\
\text { cuidador, falta de apoio } \\
\text { de outros familiares, o } \\
\text { grau de dependência } \\
\text { e o desgaste físico } \\
\text { e psicológico } \\
\text { são os principais } \\
\text { fatores geradores } \\
\text { de sobrecarga dos } \\
\text { familiares. }\end{array}$ \\
\hline $\begin{array}{l}\text { Legenda: } \\
\star \text { *b-on } \\
{ }_{\star * *} \text { Pubmed } \\
* * * \text { EBSCO host }\end{array}$ & 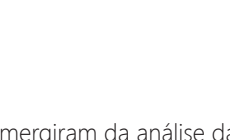 & 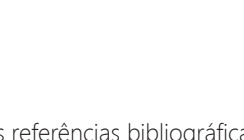 & & & & \\
\hline
\end{tabular}

\section{DISCUSSÃO}

De acordo com a nossa análise, denota-se uma escassez de estudos sobre as implicações desta doença nos restantes membros da família, bem como sobre a adaptação das famílias e o uso de recursos de saúde face à doença. Por outro lado, existe uma abundância de estudos nacionais e internacionais que abordam o impacto no cuidador principal, sendo considerado o elemento representativo de toda a unidade familiar. O estudo da temática tem suscitado interesse dos investigadores pelo uso de diversas metodologias: qualitativa, quantitativa e mista; revisão da literatura, sistemática e integrativa. Nos estudos primários, pela complexidade dos fenómenos associados ao aparecimento desta doença, recorre-se maioritariamente a métodos qualitativos e mistos. As revisões sistemáticas da literatura encontradas revelam um avanço significativo recente no estudo do tema. De acordo com os estudos analisados verificamos que a sobrecarga dos cuidadores é o indicador em saúde mais avaliado. As temáticas emergentes dos diferentes estudos analisados são o processo de cuidar; os sentimentos ambíguos decorrentes do processo; o desgaste biopsicossocial do cuidador e a sua imposição para esta função e a dependência da pessoa cuidada. Para os autores ${ }^{(29)}$, os cuidadores não apresentam mecanismos de resolução adequados para lidar diariamente com a pessoa dependente. A resposta face aos eventos críticos pode não ser a mais adequada, devido aos elevados níveis de desgaste físico, emocional e psicológico a que estão constantemente sujeitos. Vários estudos demonstram que a responsabilidade dos cuidados domiciliários recai frequentemente sobre um único membro da família, gerando sobrecarga com consequências físicas e psíquicas como stress, depressão e ansiedade ${ }^{(37-38-39)}$ assim como, autoabandono com negligencia nas atividades de lazer, vida social, familiar e afetiva. As dificuldades económicas decorrentes deste 
processo também geram diminuição da qualidade de vida com elevado risco de doença grave. Os autores $^{(40)}$ referem que os cuidadores possuem ajuda dos outros membros da família, no entanto, a sobrecarga da responsabilidade é contínua resultando em cansaço e elevados níveis de stress. Um estudo realizado(41) demonstra diversas dificuldades relatadas pelos familiares, referindo que apesar das mudanças geradas pela dependência do familiar, os cuidados prestados são realizados por amor, gratidão e respeito, de forma, a proporcionar uma melhor qualidade de vida.

Torna-se importante a colaboração dos profissionais de saúde a fim de promoverem a corresponsabilidade de todos os elementos do agregado familiar, de modo a distribuírem as tarefas necessárias. Deste modo, torna-se importante o acompanhamento regular dos cuidadores por uma equipa multidisciplinar, para identificar as necessidades da família( ${ }^{(41-42)}$ prevenindo o desgaste do cuidador principal. As alterações biopsicossociais vividas pelos familiares cuidadores evidenciam diminuição do orçamento familiar, e agravamento do estado físico e psicológico gerados pela sobrecarga. Os autores ${ }^{(18)}$ referem a existência de medidas atenuantes que podem contribuir para o alívio do stress diário com a realização frequente da prática de yoga, a meditação, a religiosidade e o relacionamento positivo entre a família, o idoso e o cuidador. A indicação de uma má qualidade de vida por parte do cuidador, pode ser associada ao desenvolvimento de um quadro de stress. O termo stress está relacionado a alterações, problemas, dificuldades ou efeitos adversos que desencadeiam desajustes físicos e psicológicos relativos a uma questão específica(43). O presente trabalho aponta para a necessidade de elaborar mais pesquisas no âmbito do impacto das demências nos diferentes elementos do agregado familiar, bem como na dinâmica do agregado e não restringir os estudos maioritariamente ao cuidador principal (Figura 2).

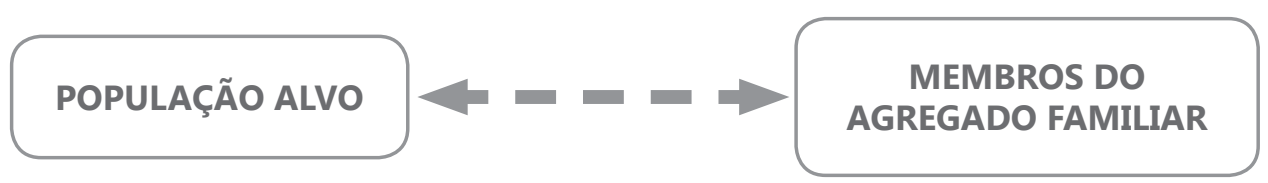

Figura 2 - Focos de atenção estudados mediante a população alvo em estudo.

Através da realização deste trabalho verificamos que a maioria dos estudos internacionais abordam o cuidador principal como figura representativa de toda a unidade familiar. A família é a principal responsável pela prestação dos cuidados de saúde aos diferentes elementos do agregado familiar, com o objetivo primordial de manter a autonomia e a dignidade da pessoa doente. Regularmente este papel é assumido por um dos elementos da família, no qual recai toda a responsabilidade dos cuidados, sem a ajuda dos outros elementos do agregado traduzindo-se em alterações significativas na dinâmica familiar.

\section{LIMITAÇõES DO ESTUDO}

Apesar dos resultados interessantes que o presente estudo concedeu, apresenta algumas limitações. A investigação têm-se debruçado sobretudo nos cuidadores informais, nos profissionais de saúde, nos pacientes e díade cuidador/paciente. Contudo, dado o reconhecimento de natureza contínua dos cuidados, emerge como especial foco de atenção a gestão das necessidades, preocupações, insegurança, medos, identificação de recursos e estratégias para o cuidar relativamente aos cuidadores informais. Para estudos futuros é importante abordar os restantes membros do agregado familiar, de modo, a perceber quais são as repercussões a nível social, emocional, económico e espiritual que surgem com o aparecimento da demência num dos seus membros. 


\section{CONSIDERAÇÕES FINAIS}

Os resultados demonstram que os cuidadores de pessoas com demência apresentam elevados níveis de sofrimento com a evolução da doença do seu familiar. Apresentam dificuldade em lidar com as alterações comportamentais, elevados níveis de sobrecarga e dificuldade na gestão do tempo e das emoções. Os estudos mostram que o impacto desta doença na vida dos cuidadores se baseia na sobrecarga emocional, stress diário e agravamento da sintomatologia depressiva.

Os enfermeiros possuem um papel fundamental na capacitação dos cuidadores familiares, no entanto, consideramos que o foco de atenção deve abranger os restantes elementos do agregado familiar, de modo, a minimizar o desgaste físico e emocional decorrente do processo de cuidar centrado num elemento da família, o cuidador principal. Assim, proporcionar programas educativos para os distintos elementos do agregado familiar contribui para uma prestação de cuidados mais eficaz, prevenindo o surgimento de doenças e a manutenção da qualidade de vida de todos os intervenientes.

Através dos resultados obtidos, podemos verificar que o impacto do surgimento desta doença nos restantes elementos do agregado familiar não tem sido alvo de estudo, uma vez que, a maioria dos estudos abordam as repercussões decorrentes do cuidar no cuidador principal. É imprescindível para a qualidade dos cuidados de saúde prestados identificar as necessidades, as dificuldades e as estratégias mais adequadas nos diferentes elementos do agregado familiar. Isto possibilita à pessoa doente um cuidado mais digno sem deixar de pensar no suporte familiar para o cuidador principal.

\section{REFERÊNCIAS}

1. HelpAge International. Ageing in the 21st Century: A Celebration and a Challenge. 2017; Accessed in: http://www.helpage.org/resources/ ageing-in-the-21st-century-a-celebration-and-a-challenge/

2. Sequeira C. Cuidar de idosos com dependência física e mental. 2018; Lidel $2^{\mathrm{a} e d i c ̧ a ̃ o . ~}$ Edições Técnicas.

3. Figueiredo M, Martins M. Avaliação Familiar: Do Modelo de Calgary de Avaliação da Família aos focos da prática de enfermagem. Ciência, Cuidado e Saúde. 2013; 9 (3): 552-559

4. Mendes C, Santos A. The care in Alzheimer's disease: social representations of family caregivers. 2016; Saúde Soc. São Paulo, v.25 (1): 121-132. DOI:10.1590/ S0104-12902015142591

5. Sousa L, Patrão M, Vicente H. Famílias e Envelhecimento: O Último Estádio Do Ciclo De Vida. In C. Paúl, \& O. Ribeiro, Manual de Gerontologia. Aspetos biocomportamentais, psicológicos e sociais do envelhecimento. 2012; 255-271. Lisboa: Lidel - edições técnicas Lda.

6. Peters MDJ, Godfrey C, McInerney P, Baldini Soares C, Khalil H, Parker D. Chapter 11: Scoping Reviews. In: Aromataris E, Munn Z (Editors). Joanna Briggs Institute Reviewer's Manual. The Joanna Briggs Institute, 2017.

7. Armstrong R, Hall BJ, Doyle J, Waters E. Cochrane Update 'Scoping the scope' of a cochrane review. J Public Health. 2011; 33(1):147-50.

8. Fernandes C, Margareth A, Martins, MM. Family caregivers of dependent elderly: same needs, different contexts - a focus group analysis. Geriatr Gerontol Aging. 2018; 12(1):31-7.

9. Correia A, Teixeira J, Oliveira, M, Magalhães S, Gomes V, Novais S. CuidaOAZ: Satisfação dos cuidadores familiares de idosos dependentes em oliveira de azeméis. Revista de Investigação \& Inovação em Saúde, 2018; 1(2): 13-22. 
10. Oliveira I, Oliveira C, Santos A, Carvalho A, Gallotti F. Impacto da Doença de Alzheimer para o familiar cuidador: Uma revisão integrativa. International nursing congresso. Theme: Good practices of nursing representations In the construction of society. 2017.

11. Farina N, Page T, Daley S, Brown A, Bowling A, Basset T, Livingston G, Knapp M, Murray J, Banerjee S. Factors associated with the quality of life of family carers of people with dementia: A systematic review. Alzheimer's \& Dementia. 2017; (13): 572-581

12. Chen CK, Clayton K, Chodosh J. The Relationship Between "What We Believe" and "How We Care" Among Daughters Caring for a Parent With Dementia. American Journal of Alzheimer's Disease \& Other Dementias ${ }^{\circledR}$ 2017, Vol. 32(2): 90-95. DOI: $10.1177 / 1533317517689875$

13. Bjorge H, Kvaal K, Smastuen MC, Ulstein I. Relationship Quality and Distress in Caregivers of Persons With Dementia: A Cross-Sectional Study. American Journal of Alzheimer's Disease \& Other Dementias ${ }^{\circledR}$ 2017, Vol. 32(3): 157-165. DOI: $10.1177 / 1533317517691121$.

14. Weisman A, Weintraub MJ, Gurak K, Maura J, Andino AM, Brown CA. Free will perceptions, religious coping, and other mental health outcomes in caregivers of individuals with dementia. Journal of Religion, Spirituality \& Aging Oct-Dec. 2017; Vol. 29 (4): 226-247.

15. Cesário VAC, Leal MCC, Marques APO, Claudino KA. Estresse e qualidade de vida do cuidador familiar de idoso portador da doença de Alzheimer. Saúde debate rio de janeiro. 2017; V. 41 (112): 171-182.

16. Marins AMF, Silva JO. Comportamento do idoso com doença de Alzheimer: reflexões à luz do interacionismo simbólico. Revista de Enfermagemdo Centro Oeste Mineiro. 2017; 7: e2484DOI: 10.19175/recom. 7i0.2484

17. Baggio C, Pedrosa AF. O impacto da doença de alzheimer no cuidador: reflexões sobre degeneração e desequilíbrio familiar. Revista Conversatio Volume 2 - Número 2 - Edição Especial - Jan/Jun. 2017; ISSN 2525-9709

18. Leite BS; Camacho ACLF; Queiroz RS. A vulnerabilidade dos cuidadores de idosos com demência: revisão integrativa. Rev Fund Care Online. 2017; 9(3): 888-892. DOI: http://dx.doi.org/10.9789/2175- 5361.2017.v9i3.888-892

19. Folle AD, Shimizu HE, Naves JOS. Social representation of Alzheimer's disease for family caregivers: stressful and rewarding. Rev Esc Enferm USP. 2016; 50(1):79-85.

20. Zauszniewski JA, Lekhak N, Burant CJ, Underwood PW, Morris DL. Resourcefulness Training for Dementia Caregivers: Establishing Fidelity. Western Journal of Nursing Research. 2016; 38(12):1554-1573. http://doi.org/10.1177/0193945916655798

21. Manzini C, Brigola AG, Pavarini SCI, Vale FAC. Fatores associados à resiliência de cuidador familiar de pessoa com demência: revisão sistemática. Rev. BRas. Geriatr. Gerontol., Rio de Janeiro. 2016; 19(4):703-714

22. Kupeli N, Leavey G, Moore K, Harrington J, Lord K, King M, Nazareth I, Sampson EL, Jones, L. Context, mechanisms and outcomes in end of life care for people with advanced dementia. BMC Palliative Care. 2016; 15:31 DOI 10.1186/ s12904-016-0103-x

23. Chiao CY, Wu HS, Hsiao CY. Caregiver burden for informal caregivers of patients with dementia: A systematic review. Int Nurs Ver. 2015; 62: 340-350.

24. Alvira MC, Risco E, Cabrera E, Farré M, Hallberg IR, Bleijlevens MHC, Meyer G, Koskenniemi J, Soto M, Zabalegui A. On behalf of the Right Time Place Care Consortium. The association between positive-negative reactions of informal caregivers of people with dementia and health outcomes in eight European countries: a cross-sectional study. Journal of Advanced Nursing. 2014; 00(0), 000-000. doi: 10.1111/jan.12528 
25. Gilson GC, Teixeira OS, Novak JC, Galhardi M. Health impacts of the family caregiver of people with alzheimer's disease: a literature review. Revista Ciência e Estudos Acadêmicos de Medicina - Número 3.Universidade do Estado de Mato Grosso - UNEMAT (Cáceres). 2015: 57-71.

26. Matsushita $M$, Ishikawa $T$, Koyama $A$, Hasegawa $N$, Ichimi N, Yano $H$, Hashimoto $M$ Fujii N, Ikeda M. Is sense of coherence helpful in coping with caregiver burden for dementia? Psychogeriatrics. 2014; vol. 14: 87-92

27. Gibson R, Gander P, Jones L. Understanding the sleep problems of people with dementia and their family caregivers. Dementia. 2014; Vol. 13(3): 350-365

28. Crellin EN, Orrell M, McDermott O, Charlesworth G. Aging \& Mental Health. 2014; Vol. 18 (8): 954-969, http://dx.doi.org/10.1080/13607863.2014.915921

29. Seima MD. Relação no cuidado entre o cuidador familiar e o idoso com Alzheimer. Revista Brasileira de Enfermagem. 2014; 2ed., vol. 67: 233-240.

30. Bremenkamp MG, Rodrigues LR, Lage RR, Laks J, Cabral HWS, Morelato RL. Sintomas neuropsiquiátricos na doença de Alzheimer: frequência, correlação e ansiedade do cuidador. Rev. Bras. Geriatr. Gerontol., Rio de Janeiro. 2014; 17(4):763-773.

31. Llanque S, Savage L, Rosenburg N, Honor's B, Caserta M. Concept Analysis: Alzheimer's Caregiver Stress. Nursing Forum. 2014; 51(1): 21-31. http://doi. org/10.1111/nuf.12090

32. Neumann S, Dias C. Alzheimer's disease: what changes in the lifestyle of family caregiver? Revista Psicologia e Saúde. 2013; v. 5 (1): 10-17.

33. Rocha $B$, Pacheco J. Idoso em situação de dependência: estresse e coping do cuidador informal. Acta Paul Enferm.2013; 26(1):50-6.

34. Andrade CC, Brasil MC. Reconfiguração de campo do familiar cuidador do portador de alzheimer. Psicologia em Estudo, Maringá. 2013; v. 18 (4): 713-723.

35. Oliveira AP, Caldana R. As Repercussões do Cuidado na Vida do Cuidador Familiar do Idoso com Demência de Alzheimer. Saúde Soc. São Paulo. 2012; v.21 (3): 675-685.

36. Baptista BO, Beuter M, Girardon-Perlini NMO, Brondani CM, Budó MLD, Santos NO. A sobrecarga do familiar cuidador no âmbito domiciliar: uma revisão integrativa da literatura. Rev. Gaúcha Enferm., Porto Alegre (RS). 2012; 33 (1):147-56.

37. Chumbler NR, Rittman M, Van Puymbroeck M, Vogel WB, Qnin H. The sense of coherence, burden and depressive symtoms in informal caregivers during the first month after stroke. International Journal of Geriatric Psychiatry. 2004; 19 (10): 944-953.

38. 38. Ducharme F, Lebel P, Bergman H. Vieillissement et soins, I'urgence d'offrir des services de santé integrés aux familles du XXI siècle. Ruptures, revue transdiciplinaire en santé. 2001; 8(2): 110-121.

39. 40. Matos PCB, Decesaro MN. Características de idosos acometidos pela doença de Alzheimer e seus familiares cuidadores principais. Rev. Eletr. Enf., Goiânia. 2012; v. 14 (4): 857-65.

40. 41. Andrade LM. Suporte familiar ao cuidador da pessoa com Doença de Alzheimer. Revista Kairós Gerontologia. 2014; 4 ed. vol.17: 275-295.

41. 42. Pestana LC, Caldas CP. Cuidados de enfermagem ao idoso com Demência que apresenta sintomas comportamentais. Rev. Bras. Enferm. 2009; 62(4).

42. 43. Garces SB, Krug M, Hansen D, Brunelli A, Costa F, Rosa C, Bianchi P, Mattos C, Seibel R. Avaliação da resiliência do cuidador de idosos com Alzheimer. Rev. Bras. Geriatr. Gerontol., Rio de Janeiro. 2012; 15 (2): 335-352. 